
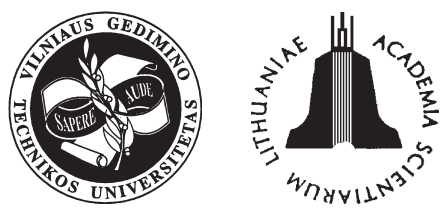

ISSN 1648-4142 TRANSPORT

www.transport.vtu.lt

TRANSPORT - 2005, Vol XX, No 6, 265-273

\title{
ACCIDENTAL EXPLOSIONS ON THE RAILWAY: SIMULATION-BASED PREDICTION OF DAMAGE TO NEARBY BUILDINGS
}

\author{
Egidijus R. Vaidogas \\ Dept of Reinforced Concrete Structures, Vilnius Gediminas Technical University, \\ Sauletekio al.11, LT-10223Vilnius, Lithuania,e-mail:erv@st.vtu.lt
}

Received 7 July 2005; accepted 30 September 2005

\begin{abstract}
A procedure for estimating potential damage to buildings induced by accidental explosions on the railway is developed. By the damage failures of nearby structures due to actions generated by the accidental explosions are meant. This damage is measured in terms of probabilities of potential failures caused by explosions. The estimation of the damage probabilities is based on stochastic simulation of railway accidents involving an explosion. The proposed simulation-based procedure quantifies epistemic (state-of-knowledge) uncertainties in the damage probabilities. These uncertainties are expressed in terms of Bayesian prior and posterior distributions. The foundation of the procedure is a computer intensive method known as the Bayesian bootstrap. It is used for approximating the posterior distributions of damage probabilities. The application of the Bayesian bootstrap makes the proposed procedure highly automatic and convenient for assessing structures subjected to the hazard of the accidental actions. In addition, it can be used for specifying safe distances between the railway and nearby buildings. Structures of these buildings can be designed for tolerable probabilities of failures induced by accidental explosions.
\end{abstract}

Keywords: railway, accident, explosion, Monte Carlo simulation, bootstrap, Bayesian bootstrap, damage.

\section{Introduction}

Accidental explosions (AEs) on the railway are dangerous, generally large-scale phenomena. Accidents involving such phenomena include severe damage to buildings and non-structural property. A selection of such accidents on the railway is described in the books $[1,2]$. Industrial activities require rail transportation of explosives and such combustible materials as liquefied gases. They constitute an inevitable potentiality of AEs. Typical AEs are bursting of explosives and physical phenomena are called the unconfined vapour cloud explosions (UVCEs) and boiling liquid expanding vapour explosions (BLEVEs). On the other hand, AEs on the railway are generally rare, unexpected, and difficult-to-predict phenomena. Actions induced by AEs are uncertain and so is the potential mechanical damage from AEs. Prediction of this damage requires proper dealing with uncertainties related to both blast loading from AEs and response of structures to AEs. In addition, general knowledge and statistical data on AEs are usually limited. The presence of considerable uncertainties generates a need to apply probabilistic methods to assessing the damage from AEs.
This paper develops a procedure for estimating potential damage to buildings induced by accidental explosions on the railway. By the damage failures of nearby structures caused by actions generated by AEs are meant. This damage is measured in terms of probabilities of potential failures caused by AEs. The estimation of the damage probabilities is based on stochastic simulation of railway accident involving an explosion. The proposed simulation-based procedure quantifies epistemic (state-of-knowledge) uncertainties in the damage probabilities. These uncertainties are expressed in terms of Bayesian prior and posterior distributions. The foundation of the procedure is a computer intensive method known as the Bayesian bootstrap. It is used for approximating the posterior distributions of damage probabilities. The application of the Bayesian bootstrap makes the proposed procedure highly automatic and convenient for assessing structures subjected to the hazard of accidental actions. In addition, it can be used for specifying safe distances between the railway and nearby buildings. Structures of these buildings can be designed for tolerable probabilities of failures induced by AEs. 


\section{Methodological background}

The prediction of potential damage to structures from rare AEs on the railway can be formulated as a problem of statistical inference. As long as the damage probability serves as a damage measure, its estimation amounts to statistical estimation of mean value. However, this estimation is far from trivial. Limited knowledge and uncertainties related to AEs render the application of classical statistics of impossible. A natural approach applies methods of the Bayesian statistical theory. They do not break down in the situation of the limited knowledge about AEs. The damage probabilities can be estimated by combining Bayesian inference with the methods of structural reliability analysis (SRA). Such a combination is well known in the field of SRA. Nevertheless, the author's experience suggests that standard techniques of Bayesian updating are not fully suited to the estimation of damage probabilities. These techniques are too general to take account of the specificity of prediction of AEs and damage from them.

Standard Bayesian updating can be enhanced by a computer intensive method of applied statistics known as the bootstrap. It belongs to methods of applied statistics which have been facilitated by the continuing development in computer technology [3]. These methods allow the analyst to assess the statistical accuracy of probability estimation by exploiting the power of the computer. The term "bootstrap" is usually understood as a means, a computationally intensive data-based simulation method for frequentist or Fisherian inference [4]. In particular, the bootstrap is understood as a computer intensive method for assigning frequentist measures of accuracy to point estimates. The merit of the bootstrap is that it relieves the analyst from having to do complex mathematical derivations [5]. Sometimes, it gives an answer where no analytical answer can be found. In addition, the bootstrap enjoys the advantage of being completely automatic (requires no theoretical calculations).

Strictly speaking, the bootstrap is not a purely frequentist means because there is a Bayesian form of the bootstrap or the Bayesian bootstrap. The latter term was introduced by Rubin [6] in 1981 who first found the connection of the bootstrap with Bayesian inference. Bayesian bootstrap is a specialized application of the bootstrap intended for simulating the posterior distribution of a parameter [3]. Rubin [6] suggested a nonparametric Bayesian bootstrap for solving this problem. In recent years, there have been many computer-intensive methods proposed to approximate posterior distributions in the Bayesian statistical analysis (e.g. [7-11]).
The Bayesian form of the bootstrap offers the scope for its application to quantitative risk assessment (QRA), as methods of QRA are substantially based on the Bayesian statistical theory (e.g. [12]). The standard models of QRA, fault trees and event trees, require Bayesian inference to specify epistemic uncertainty distributions for such model parameters as probabilities of basic events in fault trees and branching probabilities in event trees (e.g. [13]). These QRA models are a potential field of application of the Bayesian bootstrap to QRA.

QRAs often deal with large-scale accidents which may include AEs. Mechanical actions imposed on structures by AEs are called in terms of structural engineering of the explosive actions $[14,15]$. A potential damage to structures due to AEs can be assessed by estimating probabilities of foreseeable damage events. Formally, these probabilities can be handled by means of the bootstrap within the pure frequentist framework [16]. However, QRA provides consistent means for dealing with considerable uncertainties related to AEs and, sometimes, response of structures to AEs. QRA means allow quantifying epistemic uncertainties in probabilities of the events in question in the form of prior and posterior distributions (e.g. [17]). The Bayesian bootstrap is suitable to utilize attractive features of data resampling techniques. It allows applying these techniques to effective Bayesian updating within QRA which considers AEs and potential damage from them. In particular, the Bayesian bootstrap can be applied to approximating posterior distributions of damage probabilities.

The present paper considers practical application of the Bayesian bootstrap to QRA focused on assessing damage to structures. It is used for Bayesian inference based on two sources of information:

- prior knowledge existing mainly in the form of mathematical models and historical data suitable for approximate prediction of AE characteristics and

- new information consisting of a small-size sample of measurements of $\mathrm{AE}$ characteristics which are highly relevant to an exposure situation (a situation in vicinity of the railway where the structure under investigation stands and a potential $\mathrm{AE}$ can occur).

The application of the Bayesian approach falls within the general approach to QRA known as classical Bayesian approach [13]. It is shown how to utilize the prior knowledge to specify prior distribution of a damage probability. Then it is demonstrated how to approximate its posterior distribution by means of the Bayesian bootstrap when the new sample of AE characteristics becomes available. 


\section{Probability of explosive damage}

Damage to a structure from AE can be represented by a finite set of $n_{d}$ random events $D_{i}(i=1,2$, $\left.\ldots, n_{d}\right)$, each standing for a foreseeable and specific mechanical damage phenomenon. If the damage is assessed in the context of QRA, probabilities of $D_{i} \mathrm{~s}$ can be grouped together to establish a risk profile related to a particular $\mathrm{AE}$ and a specific exposure situation [18].

The probability of damage $D_{i}$ due to AE can be expressed in the form

$$
P\left(D_{i} \mid A E\right)=\int_{\text {all } \boldsymbol{y}} P\left(D_{i} \mid \boldsymbol{y}\right) \mathrm{d} F_{\boldsymbol{Y}}(\boldsymbol{y})=E\left(P\left(D_{i} \mid \boldsymbol{Y}\right)\right),
$$

where $D_{i}$ is the random event of damage; $E A$ is the random event of imposition of $\mathrm{AE}$ with any characteristics; $\boldsymbol{Y}$ is the random vector of $\mathrm{AE}$ characteristics; $\boldsymbol{y}$ and $F_{\boldsymbol{Y}}(\boldsymbol{y})$ are the value of $\boldsymbol{Y}$ and its distribution function (d.f.), respectively.

The definition (1) is based of fragility function $P\left(D_{i} \mid \boldsymbol{y}\right)$ relating particular value of AE characteristics, $\boldsymbol{y}$, to probability of damage event $D_{i}$. Fragility function is an often-used tool for describing the response of structures to extreme actions (e.g. [19, 20]).

The fragility function can be expressed as some function $p_{i}(\cdot)$ which relates probability of $D_{i}$ to $\boldsymbol{y}$ and thus takes on probability values, namely, $p_{i}(\boldsymbol{y})=P\left(D_{i} \mid \boldsymbol{y}\right)$. This function allows introducing a random variable $\tilde{P}$ defined as a function of the random vector $\boldsymbol{Y}$, namely,

$$
\tilde{P}=p_{i}(\boldsymbol{Y})=P\left(D_{i} \mid \boldsymbol{Y}\right) \text {. }
$$

A mean value of $\tilde{P}$ can be denoted by $\mu$ and expressed as

$$
\mu=E(\tilde{P})=E\left(P\left(D_{i} \mid \boldsymbol{Y}\right)\right) .
$$

The problem is that d.f. $F_{Y}(y)$ is not known due to scarcity or irrelevance of information on characteristics of many types of AEs. However, distribution of values of $\boldsymbol{Y}$ can be approximately predicted by existing models and, in addition, data related to $Y$ can be collected by carrying out experiments which are highly relevant to the exposure situation under investigation.

\section{Knowledge available for estimating damage prob- ability}

\subsection{Prior knowledge}

The expression (3) implies that the damage probability can be expressed as uncertain distribution parameter $\mu$ amenable to Bayesian inference. The learning process involved in Bayesian inference is one of modifying the analyst's initial probability statements about distribution parameters prior to observing data to posterior knowledge incorporating both prior knowledge and the data at hand (e.g. [21]).

In a pure Bayesian analysis, the prior distribution of $\mu$ should be specified subjectively. However, the purely subjective specification does not utilize prior knowledge about many types of AEs. Such knowledge, more or less relevant to the exposure situation under investigation, is usually available for the analyst. Therefore one can make a compromise between the frequentist and Bayesian statistical analysis and specify priors for damage probabilities from the prior knowledge.

Formal means for specifying priors based on data are provided by empirical Bayes methods [22]. This paper proposes a simple heuristic approach to speci-

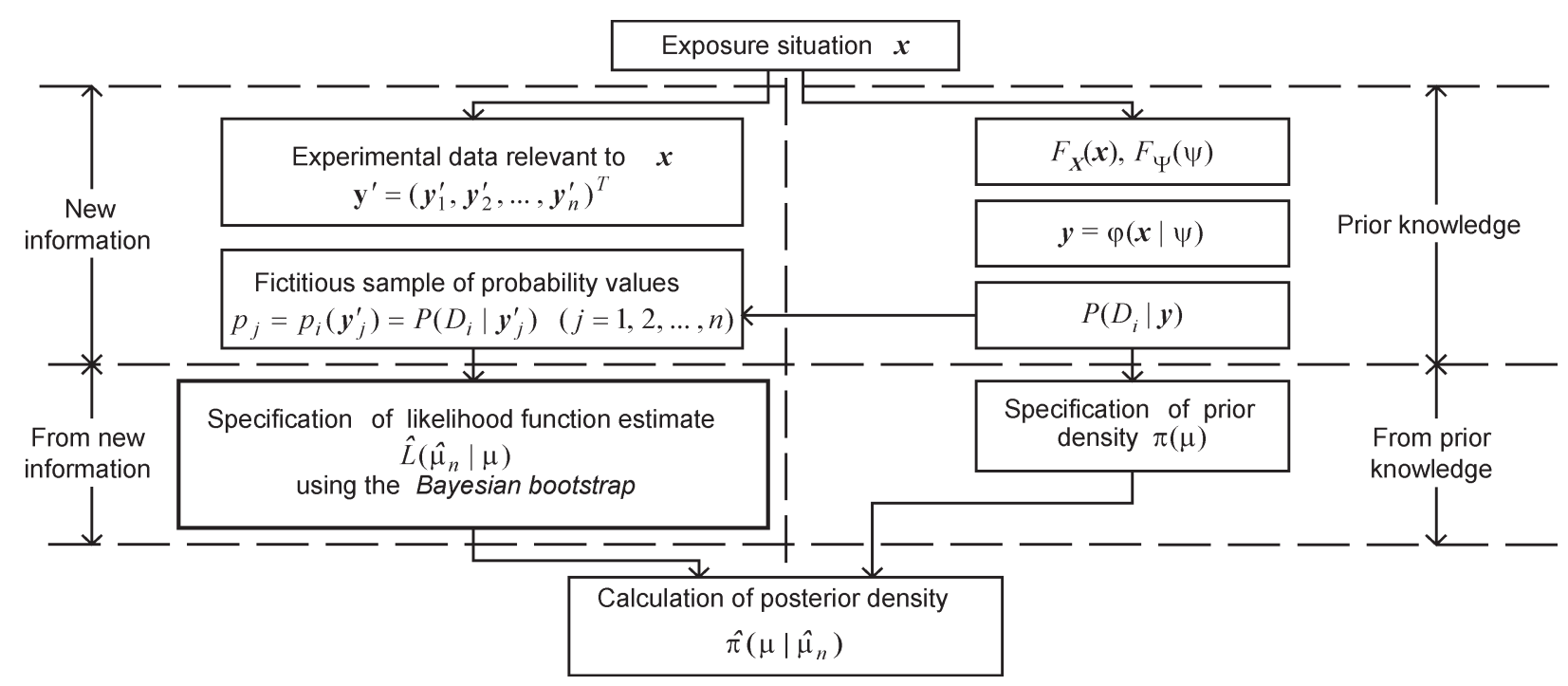

Fig 1. The role of the Bayesian bootstrap in estimating probabilities of damage to structures due to accidental actions 
fying priors from existing knowledge. The approach is based on the knowledge which is highly specific to a particular $\mathrm{AE}$ and is expressed in the form of a mathematical model $\varphi(\cdot)$ relating characteristics of exposure situation to characteristics of AE, namely,

$$
y=\varphi(x \mid \psi),
$$

where $\boldsymbol{x}$ is the vector describing characteristics of exposure situation in which AE can occur; $\boldsymbol{\psi}$ is the vector used to express epistemic uncertainties in those parameters of $\varphi(\cdot)$ which are uncertain in epistemic sense. The exposure situation represented by $x$ may be uncertain in the stochastic sense and this can be modeled by a random vector $\boldsymbol{X}$ with aleatory d.f. $F_{X}(\boldsymbol{x})$.

A part of prior knowledge should be represented by the fragility function $p_{i}(\boldsymbol{y})$ which can be established for an exposed structure by methods of the structural reliability analysis (e.g. [23]). Thus the fragility function $p_{i}(\cdot)$ together with the model $\varphi(\cdot)$ form the main part of the prior knowledge, the structure of which is shown Fig 1.

\subsection{New information}

The need to apply Bayesian inference to estimate the damage probability $P\left(D_{i} \mid A E\right)$ may stem mainly from partial irrelevance of the prior knowledge to a particular exposure situation. The configuration of a structure exposed to $\mathrm{AE}$ as well as the accident capable of inducing AE may be unique by a large margin and so may not fit in the prior knowledge. The source of the partial irrelevance may lie in

- the structure of model $\varphi(\cdot)$ and/or

- the data used to fit d.f. $F_{X}(x)$ and to estimate parameters of $\varphi(\cdot)$, that is, components of $\boldsymbol{\psi}$.

The partial irrelevance may require the correction of AE prediction by experimental data which can be considered highly relevant to an exposure situation under investigation. Clearly, these experiments can be used for improving model $\varphi(\cdot)$ by, say, increasing its relevance to the exposure situation. However, the highly relevant (case-specific) data on AE characteristics $\boldsymbol{y}$ and, possibly, interaction of $\mathrm{AE}$ with the exposed structure can be used directly estimating $P\left(D_{i} \mid A E\right)$.

In theory, the amount of the case-specific data may be such that model $\varphi(\cdot)$ will no longer be needed. In practice, however, the amount of the data may be limited because experiments on AEs, especially fullscale ones, are often expensive. This may require combining of the new, case-specific data with the prior knowledge behind $\varphi(\cdot)$.

The case-specific data necessary for estimating $P\left(D_{i} \mid A E\right)$ should be gathered and represented in the form of a sample $\mathbf{y}^{\prime}=\left(\mathrm{y}_{1}^{\prime}, \mathrm{y}_{2}^{\prime}, \ldots, \mathrm{y}_{n}^{\prime}\right)^{T}$ containing experimental observations of $\mathrm{AE}$ characteristics. Clearly, each experiment in a series yielding sample $\mathbf{y}^{\prime}$ should imitate a potential accident and sample $\mathbf{y}^{\prime}$ itself should posses the property called by statisticians the "representativeness" (e.g. [24]). Although this property of $\mathbf{y}^{\prime}$ is very important, a detailed discussion about how to ensure the representativeness is out of the scope of this paper. In subsequent discussion, it is assumed that sample $\mathbf{y}^{\prime}$ possesses this property.

Given sample $\mathbf{y}^{\prime}$ and a fragility function of interest, $p_{i}(y)$, one can simplify estimating $P\left(D_{i} \mid A E\right)$ by introducing fictitious sample $\boldsymbol{p}=\left(p_{1}, p_{2}, \ldots, p_{n}\right)^{T}$, each component of which is calculated by $p_{j}=p_{i}\left(\mathrm{y}_{j}^{\prime}\right)=P\left(D_{i} \mid \mathrm{y}_{j}^{\prime}\right) \quad(j=1,2, \ldots, n)$. In this way the problem of estimating $P\left(D_{i} \mid A E\right)$ is made less complicated by switching from an multi-dimensional analysis to a one-dimensional case. Then the components $p_{j}$ of $\boldsymbol{p}$ can be treated as realizations of random variable $\tilde{P}$ defined by (2).

Expensiveness of experiments on AEs may cause that size $n$ of sample $\mathbf{y}^{\prime}$ will be too small to apply the methods of classical statistics for estimating $P\left(D_{i} \mid A E\right)$. In addition, experiments on AE may be unique and a series of them resulting in $\mathbf{y}^{\prime}$ may be carried only once. This implies that the procedure of Bayesian updating using $\mathbf{y}^{\prime}$ will be a single act, rather than a more or less constant process.

\section{Use of the Bayesian bootstrap}

\subsection{Specifying of prior for damage probability}

Epistemic uncertainties related to parameters $\psi$ of model $\varphi(x \mid \psi)$ can be expressed introducing random vector $\boldsymbol{\Psi}$ with d.f. $F_{\boldsymbol{\Psi}}(\boldsymbol{\psi})$. Then replacing of $\boldsymbol{Y}$ in (4) by random function $\varphi(\boldsymbol{X} \mid \boldsymbol{\Psi})$ will yield another random variable

$$
\begin{aligned}
& \widetilde{M}=m(\Psi)=E_{\mathrm{X}}\left(p_{i}(\varphi(\mathrm{X} \mid \Psi))\right) \\
& =\int_{\text {all x }} P\left(D_{i} \mid \varphi(\mathrm{x} \mid \Psi)\right) \mathrm{d} F_{\mathrm{X}}(\mathrm{x}),
\end{aligned}
$$

where $m(\cdot)$ denotes some function relating $\widetilde{M}$ to $\boldsymbol{\Psi}$. A value $\mu$ of $\widetilde{M}$ is the damage probability at given $\psi$, namely, $E_{\mathrm{X}}\left(P\left(D_{i} \mid \varphi(\mathrm{X} \mid \psi)\right)\right)$. The density of $\widetilde{M}$ denoted, say, by $\pi(\mu)$ can be used as prior quantifying epistemic uncertainty in damage probability $P\left(D_{i} \mid A E\right)$ (Fig 2).

The source of the epistemic uncertainty may not necessarily be only on the side of model $\varphi(x \mid \psi)$ used to predict $\mathrm{AE}$ characteristics. Epistemic uncertainty can also be related to values of fragility function $p_{i}(y)$ at given $\boldsymbol{y}$. However, this part of epistemic uncertainty can be handled in the framework of (5) and is left out for brevity. 


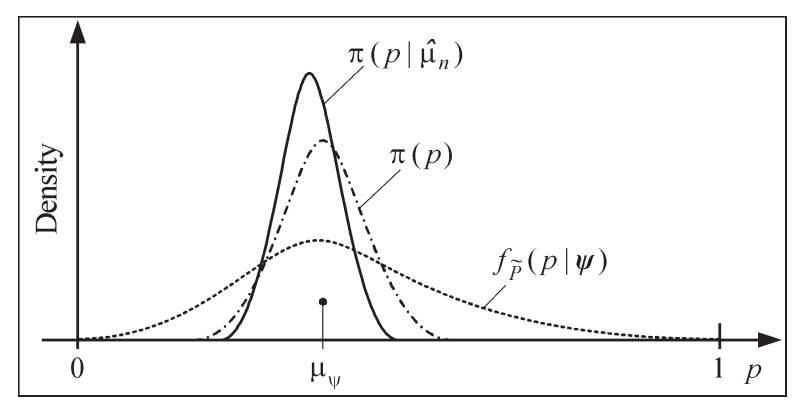

Fig 2. Schematic representation of densities related to probabilistic damage assessment in Bayesian context: $f_{\tilde{P}}(p \mid \psi)=$ density of random variable $\widetilde{P}=p_{i}(\varphi(x \mid \psi))$ with mean $\mu_{\psi} ; \pi(p)=$ prior distribution of $\mu ; \pi\left(p \mid \hat{\mu}_{n}\right)=$ posterior distribution of $\mu$

\subsection{Updating of prior by means of the Bayesian} bootstrap

The usual Bayesian posterior has the form

$\pi(\mu \mid$ data $) \propto \mu \pi(\mu) L($ data $\mid \mu)$,

where "data" is represented eventually by samples $\boldsymbol{p}$ or $\mathbf{y}^{\prime}$. The main idea followed in the paper is to replace the usual Bayesian posterior $\pi(\mu \mid$ data $)$ by estimated posterior

$$
\hat{\pi}(\mu \mid \text { data }) \propto \pi(\mu) \hat{L}_{B}(\text { data } \mid \mu),
$$

where $\hat{L}_{B}($ data $\mid \mu)$ is estimate of the likelihood function based on bootstrap estimation of the density of pivotal quantity $\hat{\mu}_{n}-\tilde{M}$ with $\hat{\mu}_{n}=n^{-1} \sum_{j=1}^{n} p_{j}$. A possibility of such a replacement was suggested by Boos and Monahan [7].

The first step is to estimate the distribution function of data $\boldsymbol{p}$ using empirical d.f. $\hat{F}_{n}$ of $p_{j}$ 's. In the second step, a set of $B$ random samples of size $n$ from $\hat{F}_{n}$ is generated and mean $\hat{\mu}_{n b}^{\prime}$ is calculated for each sample $b(b=1,2, \ldots, B)$. From $B$ simulated estimates $\hat{\mu}_{n 1}^{\prime}, \hat{\mu}_{n 2}^{\prime}, \ldots, \hat{\mu}_{n B}^{\prime}$, one can compute kernel density estimate.

$$
\hat{k}_{B}(u)=\frac{1}{B w} \sum_{b=1}^{B} \kappa\left(\frac{u-\left(\hat{\mu}_{n b}^{\prime}-\hat{\mu}_{n}\right)}{w}\right),
$$

where $w$ is bandwidth (window width, smoothing parameter) and $k(\cdot)$ is kernel function. Since function $\hat{k}_{B}(u-\mu)$ is an estimate of the sampling density of $\hat{\mu}_{n}$ given $\mu$, likelihood function of $\hat{\mu}_{n}$ can be estimated by

$$
\hat{L}_{B}\left(\hat{\mu}_{n} \mid \mu\right)=\hat{k}_{B}\left(\hat{\mu}_{n}-\mu\right)=\frac{1}{B w} \sum_{b=1}^{B} \kappa\left(\frac{\left.2 \hat{\mu}_{n}-\mu-\hat{\mu}_{n b}^{\prime}\right)}{w}\right) .
$$

The resulting estimate of posterior of the damage probability is

$$
\hat{\pi}\left(\mu \mid \hat{\mu}_{n}\right)=C\left(\hat{\mu}_{n}\right) \pi(\mu) \hat{L}_{B}\left(\hat{\mu}_{n} \mid \mu\right),
$$

where the normalizing constant $C\left(\hat{\mu}_{n}\right)$ can be found by numerical integration.

\subsection{Practical implementation}

Practical implementation of the bootstrap-based updating procedure is relatively simple, as estimates $\hat{L}_{B}\left(\hat{\mu}_{n} \mid \mu\right)$ and $\hat{\pi}\left(\mu \mid \hat{\mu}_{n}\right)$ can be computed almost automatically. Estimate $\hat{L}_{B}\left(\hat{\mu}_{n} \mid \mu\right)$ is relatively insensitive to the choice of kernel function $k(\cdot)$ [4]. The only implementation problem is associated with the choice of bandwidth $w$ which can have considerable influence on $\hat{L}_{B}\left(\hat{\mu}_{n} \mid \mu\right)$.

A simple recipe for choosing the "best" value of $w$ seems not to be available and many authors sidestep this problem by presenting estimates like $\hat{L}_{B}\left(\hat{\mu}_{n} \mid \mu\right)$ and $\hat{\pi}\left(\mu \mid \hat{\mu}_{n}\right)$ for different values of $w$ (e.g. [11]). A criterion for the choice of $w$ should be smoothness of the estimated likelihood curve $\hat{L}_{B}\left(\hat{\mu}_{n} \mid \mu\right)$. Davison et al. [4] suggest that $w$ should be proportional to $B$. Shao and Tu [3] provide a review of approaches to choosing $w$ by means of cross validation and the bootstrap. In the present context, one can only state that further investigation is necessary to develop an algorithm for an automatic choice of $w$. Without such algorithm, updating via the Bayesian bootstrap can hardly be attractive to application in the field of structural engineering.

\section{Example: assessing explosive damage to an indus- trial building}

\subsection{Exposure situation}

Consider an industrial building constructed in the vicinity of a $500 \mathrm{~m}$ section of the railway (Fig 3). This

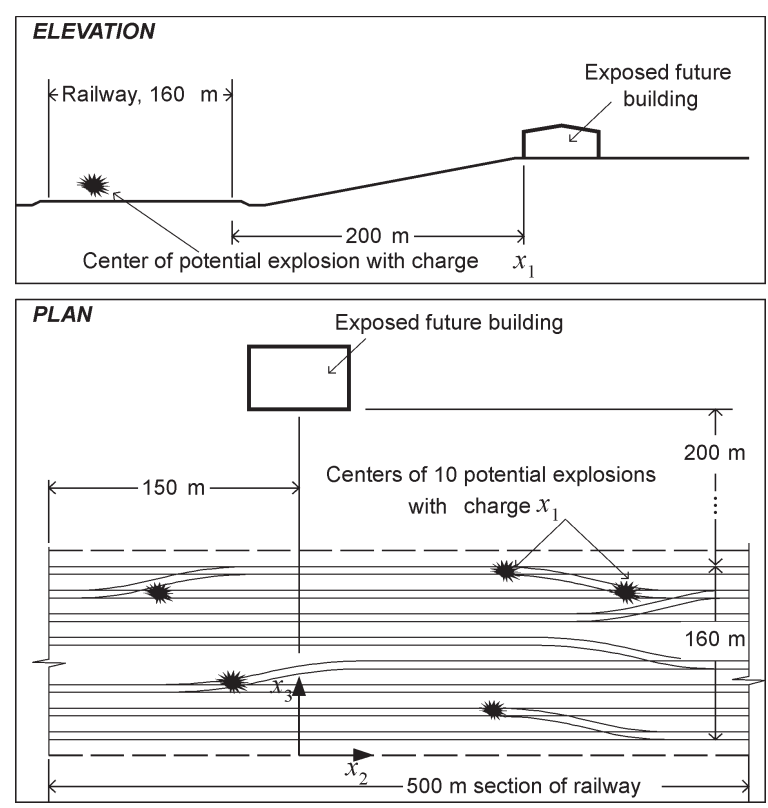

Fig 3. Exposure situation involving an accident with accidental explosion on the railway 
transportation facility is used to carry commercial explosives which can detonate in consequence of a railway accident. The accidental explosion will generate a shock front resulting in impulsive loading imposed on the building. Characteristics of this loading can be described by vector $\boldsymbol{y}=\left(y_{1}, y_{2}\right)^{T}$, where $y_{1}(\mathrm{MPa})$ and $y_{2}\left(\mathrm{MPa} \mathrm{s} / \mathrm{m}^{2}\right)$ are the peak positive overpressure and positive impulse of the incident shock front, respectively.

The damage to the building is represented by random event $D_{i}$ consisting of flexural failure or shear failure of reinforced concrete panels used for external envelope of the building. These panels should resist not only wind pressure, but also the pressure arising from the reflection of the shock front by the façade of the future building.

The exposure situation is represented by vector $\boldsymbol{x}=\left(x_{1}, x_{2}, x_{3}\right)^{T}$, where $x_{1}(\mathrm{~kg})$ is the weight of transported explosives (charge weight); $x_{2}(\mathrm{~m})$ and $x_{3}(\mathrm{~m})$ are the coordinates of the centre of accidental explosion in the coordinate system shown in Fig 3. Thus the expression $r=\left(x_{2}^{2}+\left(360-x_{3}\right)^{2}\right)^{1 / 2}$ is the standoff of the explosion to the façade and the expression $s=x_{1}^{1 / 3} r^{-1}$ is so-called inverse scaled distance $\left(\mathrm{kg}^{1 / 3} / \mathrm{m}\right)$ (inverse normalized standoff, e.g. [1]).

\subsection{Prior knowledge}

Prior knowledge is represented by the model

$$
\boldsymbol{y}=\varphi(\boldsymbol{x} \mid \psi)=\left(\varphi_{1}(\boldsymbol{x} \mid \psi), \varphi_{2}(\boldsymbol{x} \mid \psi)\right)
$$

with the model components

$$
\begin{aligned}
& y_{1}=\varphi_{1}(\boldsymbol{x} \mid \psi)=\psi_{1}\left(\psi_{1}^{\prime} s++\psi_{2}^{\prime} s^{2}+\psi_{3}^{\prime} s^{3}\right), \\
& y_{2}=\varphi_{2}(\boldsymbol{x} \mid \psi)=\psi_{2} \psi_{4}^{\prime} x_{1}^{2 / 3} r^{-1}
\end{aligned}
$$

and the vector of regression parameters

$$
\begin{aligned}
& \left(\psi_{1}^{\prime}, \psi_{2}^{\prime}, \psi_{3}^{\prime}, \psi_{4}^{\prime}\right)^{T}=\left(0,1 \mathrm{MPa} \times \mathrm{m} / \mathrm{kg}^{1 / 3} ;\right. \\
& 0,43 \mathrm{MPa} \times \mathrm{m}^{2} / \mathrm{kg}^{2 / 3} ; 1,4 \mathrm{MPa} \times \mathrm{m}^{3} / \mathrm{kg} ; \\
& \left.6,3 \mathrm{MPa} \times \mathrm{s} /\left(\mathrm{m} \mathrm{kg}^{2 / 3}\right)\right)^{T} .
\end{aligned}
$$

Components of vector $\psi=\left(\psi_{1}, \psi_{2}\right)^{T}$ are dimensionless adjustment factors (relative overpressure and relative impulse of the commercial explosive, which can detonate in consequence of the railway accident, compared to the equivalent weight of TNT explosive).

Stochastic uncertainty is related to the exposure situation and so arguments of model $\varphi(x \mid \psi)$ are expressed by random vector $\boldsymbol{X}=\left(X_{1}, X_{2}, X_{3}\right)^{T}$ with components distributed as follows $X_{1} \sim \mathrm{N}(500 \mathrm{~kg}, 30$ $\mathrm{kg}), X_{2} \sim \mathrm{U}(0 \mathrm{~m}, 160 \mathrm{~m}), X_{3} \sim \mathrm{U}(0 \mathrm{~m}, 500 \mathrm{~m})$, where $\mathrm{L}$ and $\mathrm{U}$ denote the normal distribution and the uniform distribution, respectively. The uniform distribution of $X_{2}$ and $X_{3}$ implies that the accidental explosion can occur with the same probability within $500 \mathrm{~m}$ $\times 160 \mathrm{~m}$ area of the railway. The uniform distribution of the explosion point coordinates $X_{2}$ and $X_{3}$ is considered as only the first approximation to forecasting the position of the explosion centre.

Epistemic uncertainty can be introduced into model $\varphi(x \mid \psi)$ by assuming components $\psi_{1}$ and $\psi_{2}$ of $\boldsymbol{\psi}$ to be random variables. Adjustment factors $\psi_{1}$ and $\psi_{2}$ are conventionally expressed as fixed values or deterministic function of so-called scaled distance $[25,26]$. However, the nature of explosive actions is highly random and adjustment factors may be expressed in the form of random variables $\Psi_{1}$ and $\Psi_{2}$ having epistemic distributions. In this example, lognormal distribution is assumed for random adjustment factors: $\Psi_{1} \sim \mathrm{L}(0,15842 ; 0,09975)$ with mode of 1,16 ; $\Psi_{2} \sim \mathrm{L}(0,17551 ; 0,09975)$ with the mode of 1.18 .

Further part of prior knowledge is represented by fragility function $p_{i}(\cdot)$ which relates peak positive overpressure $y_{1}$ and positive impulse $y_{2}$ to failure probability $P\left(D_{i} \mid \boldsymbol{y}\right)$. The form of $p_{i}(\boldsymbol{y})$ can be established using the results obtained by Low \& Hao [27], who investigated the reliability of RC slabs under impulsive loads. The function $p_{i}(y)$ is represented in the form

$$
p_{i}(\boldsymbol{y})=P\left(D_{i} \mid \varphi^{\prime}\left(x_{1}, x_{2}, y_{1}\right), \varphi^{\prime \prime}\left(y_{1}, y_{2}\right)\right),
$$

where $\varphi^{\prime}($.$) and \varphi^{\prime \prime}($.$) are two deterministic functions$ which relate the peak overpressure and impulse of reflected shock front to the respective characteristics of the incident shock front. The function $\varphi^{\prime}($.) is called the "reflected pressure factor versus angle of incidence" and is usually represented in the graphical form [25]. The angle of incidence of the shock front can be simply determined from the explosion point coordinates $x_{2}$ and $x_{3}$. The function $\varphi^{\prime \prime}($.$) is a simple$ formula allowing estimating the reflected impulse from incident impulse $y_{2}$ [1].

In this example, fragility function $p_{i}(y)$ is approximated by a d.f. $F\left(z_{1}, z_{2} \mid \mu_{P 1}, \mu_{P 2}, \sigma_{P 1}, \sigma_{P 2}, \rho\right)$ of bivariate normal distribution, namely,

$$
p_{i}\left(z_{1}, z_{2}\right)=F\left(z_{1}, z_{2} \mid \mu_{P 1}, \mu_{P 2}, \sigma_{P 1}, \sigma_{P 2}, \rho\right),
$$

where arguments $z_{1}$ and $z_{2}$ are defined as $z_{1}=\varphi^{\prime}\left(x_{1}, x_{2}, y_{1}\right)$ and $z_{2}=\varphi^{\prime \prime}\left(y_{1}, y_{2}\right)$ and parameters $\mu_{P 1}=3,2 \times 10^{-3} \mathrm{MPa}, \mu_{P 2}=1,45 \mathrm{MPa} \times \mathrm{s} / \mathrm{m}^{2}, \sigma_{P 1}$ $=0,64 \times 10^{-3} \mathrm{MPa}, \sigma_{P 2}=0,29 \mathrm{MPa} \times \mathrm{s} / \mathrm{m}^{2}, \rho=0,2$.

Clearly, the analyst may have uncertainties in epistemic sense related to the elements of model $p_{i}(\boldsymbol{y})$ as well as further elements of model $\varphi(x \mid \psi)$, first and foremost, vector of the regression parameters, $\left(\psi_{1}^{\prime}, \psi_{2}^{\prime}, \psi_{3}^{\prime}, \psi_{4}^{\prime}\right)^{T}$; however, these uncertainties are ignored in the present example for simplicity.

The formulas (6) and (7) are standard relations obtained by experiments on TNT. They are, strictly speaking, valid only for distant explosion of a charge positioned on the ground which forms a horizontal 
plane. In addition, the model represented by (6) and (7) assumes that the shock front generated by TNT explosion does not encounter any obstacles. A gentle slope of the ground between the railway and the exposed future building makes model $\varphi(x \mid \psi)$ only partially relevant to the exposure situation shown in Fig 3. In addition, the assumption of the uniform distribution of the explosion point coordinates $X_{2}$ and $X_{3}$ may be considered sound; however, a detailed analysis of railway traffic by means of QRA may introduce corrections in this assumption.

Despite the irrelevance of the model represented by (6) and (7), it can be used for specifying prior distribution of $P\left(D_{i} \mid A A\right)$.

\subsection{Specifying prior from distribution existing} knowledge

Prior distribution of the damage probability $P\left(D_{i} \mid A E\right)$ can be specified by propagating epistemic uncertainty related to parameters $\psi_{1}$ and $\psi_{2}$. Thus uncertainty can be transformed into uncertainty in $P\left(D_{i} \mid A E\right)$ by (5). Density $\pi(\mu)$ of random variable $\widetilde{M}$ defined by (5) will serve as prior distribution.

Density $\pi(\mu)$ can be specified by fitting it to sample $\mu_{k}\left(k=1,2, \ldots, n_{k}\right)$, the elements of which are estimates of mean values $E_{\mathrm{X}}\left(p_{i}\left(\varphi\left(\mathrm{X} \mid \psi_{k}\right)\right)\right)$ for given $\psi_{k}$. Values $\psi_{k}$ are generated from epistemic d.f. $F_{\Psi}(\psi)$ by means of stochastic (Monte Carlo) simulation. Mean values $E_{X}(\cdot)$ can also be estimated by simulation:

$$
\mu_{k}=n_{e}^{-1} \sum_{l=1}^{n_{e}} p_{i}\left(\varphi\left(\mathrm{x}_{l} \mid \psi_{k}\right)\right)
$$

where $\boldsymbol{x}_{l}$ is the value of $\boldsymbol{X}$ generated from aleatory d.f. $F_{X}(x)$. Fig 4 shows density of normal distribution fitted to generated sample $\mu_{k}(k=1,2, \ldots, 1000)$ obtained using $n_{e}=1 \times 10^{5}$.

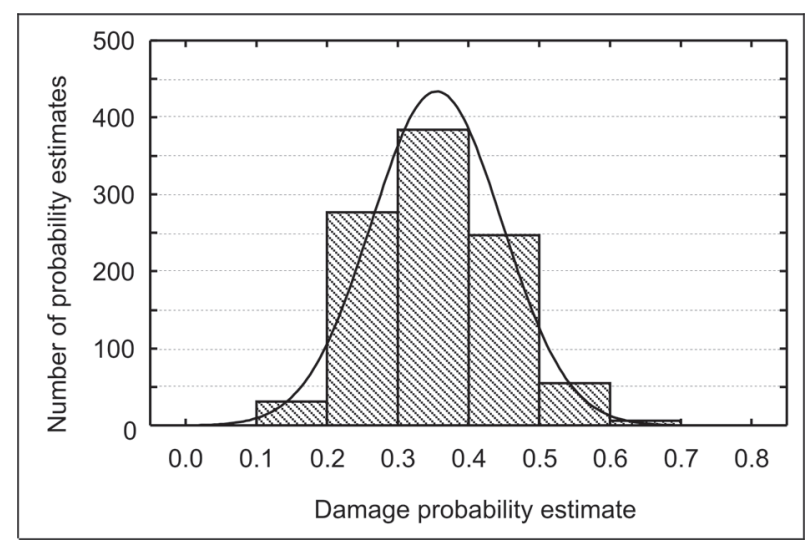

Fig 4. Histogram of sample $\mu_{k}(k=1,2, \ldots, 1000)$ and transformed density of normal distribution $\mathrm{N}(0,353$; 0,09196) fitted to the sample
Consequently, prior density $\pi(\mu)$ is specified as density of normal distribution $\mathrm{N}(0,353 ; 0,09196)$ with $90 \%$ confidence interval ]0,202; 0,504[.

\subsection{New data of a possible railway accident}

New data may be obtained by an experiment which imitates potential accidents on the railway section. A detailed analysis of traffic in the railway section can yield, say, ten potential centers of accidental explosion (Fig 3). Then a series of ten explosions can be carried out by detonating charges $x_{1}$ of the explosive under investigation in a blast measuring facility which imitates the ground surface of the exposure situation. The weight of charges, $x_{1}$, can be chosen by chance from the distribution of $X_{1}$.

The series of experiments will yield sample $\mathbf{y}^{\prime}=\left(\boldsymbol{y}_{1}^{\prime}, \boldsymbol{y}_{2}^{\prime}, \ldots, \boldsymbol{y}_{10}^{\prime}\right)^{T}$. Let us say that the results of the experiment are represented by ten pairs of measured overpressures and impulses given in Table 1.

Table 1. Observed elements $\boldsymbol{y}_{i}^{\prime}$ of sample $\mathbf{y}^{\prime}$ and computed elements $p_{j}$ of sample $\boldsymbol{p}$

\begin{tabular}{|c|c|c|}
\hline$j$ & $\mathrm{y}_{i}^{\prime}\left(\mathrm{MPa}, \mathrm{MPa} \times \mathrm{s} / \mathrm{m}^{2}\right)$ & $p_{j}$ \\
\hline 1 & $(2,06 ; 0,960)$ & 0,001674 \\
\hline 2 & $(2,99 ; 1,38)$ & 0,1484 \\
\hline 3 & $(2,68 ; 1,26)$ & 0,05482 \\
\hline 4 & $(2,73 ; 1,27)$ & 0,06283 \\
\hline 5 & $(3,41 ; 1,59)$ & 0,4286 \\
\hline 6 & $(2,66 ; 1,23)$ & 0,04543 \\
\hline 7 & $(2,74 ; 1,24)$ & 0,0545 \\
\hline 8 & $(3,29 ; 1,46)$ & 0,2861 \\
\hline 9 & $(2,92 ; 1,30)$ & 0,09814 \\
\hline 10 & $(3,82 ; 1,74)$ & 0,7022 \\
\hline
\end{tabular}

A small size of sample $\mathbf{y}^{\prime}$ is likely, as the experiment imitating the accident may be expected to be expensive and time consuming. Clearly, ten-element sample $\mathbf{y}^{\prime}$ is too-small to estimate damage probability $P\left(D_{i} \mid A E\right)$ using $\mathbf{y}^{\prime}$ alone, that is, without prior knowledge. However, this sample may be used to update the prior distribution $\mathrm{N}(0,353$; 0,09196) of $P\left(D_{i} \mid A E\right)$ specified on the basis of prior knowledge. To do this, the fragility function (8) should be applied to transform $\mathbf{y}^{\prime}$ into fictitious sample $\boldsymbol{p}$ given in Table 1.

Mean $\hat{\mu}_{10}$ of sample $\boldsymbol{p}$ is equal to 0,1882 . Sample $\boldsymbol{p}$ can be applied to estimate likelihood function $L\left(\hat{\mu}_{10} \mid \mu\right)$ and approximate posterior distribution of $P\left(D_{i} \mid A E\right)$. 


\subsection{Posterior distribution as epistemic estima- tion of damage probability}

The estimate of likelihood function, $\hat{L}_{B}\left(\hat{\mu}_{10} \mid \mu\right)$ was obtained using Gaussian kernel function $k(\cdot)$. The number of bootstrap replications, $B$, necessary to generate sample $\left(, \hat{\mu}_{n 1}^{\prime}, \hat{\mu}_{n 2}^{\prime} \ldots \hat{\mu}_{n B}^{\prime},\right)$ was taken to be equal to 1000 . The choice of $B$ was based on the rules of thumb suggested by Efron and Tibshirani [5, p. 52].

The choice of the bandwidth $w$ was not investigated in detail. Three values 0,01, 0,02, and 0,03 of $w$ were chosen manually to assess the influence of $w$ on the posterior density $\hat{\pi}\left(\mu \mid \hat{\mu}_{n}\right)$. As expected, the largest value of $w$ produced the smoothest estimate of the likelihood function. Fig 5 shows a graph of $\hat{L}_{B}\left(\hat{\mu}_{10} \mid \mu\right)$ at $w=0,03$.

Three approximations of posterior density $\hat{\pi}\left(\mu \mid \hat{\mu}_{10}\right)$ computed at three values of $w$ are shown in Fig 6 . These approximations were obtained by numerical calculation. The approximations $\hat{\pi}\left(\mu \mid \hat{\mu}_{10}\right)$ express the epistemic uncertainty related to

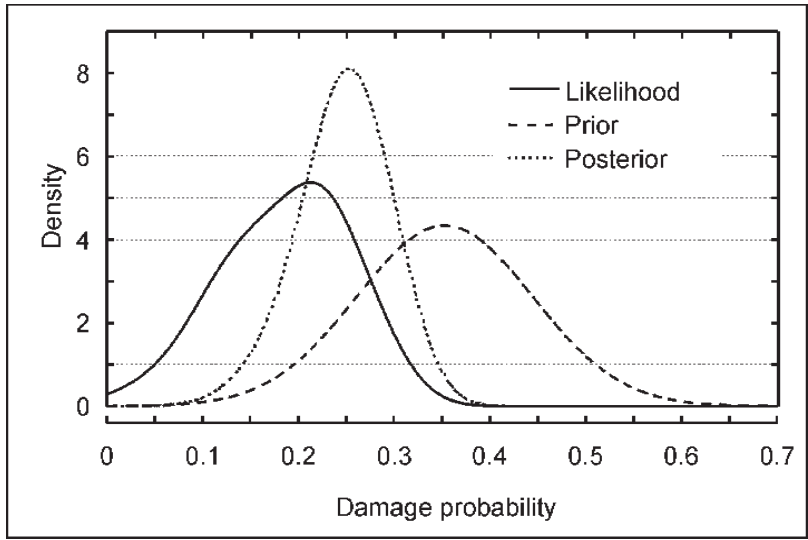

Fig 5. Graph of the likelihood function estimate $\hat{L}_{B}\left(\hat{\mu}_{10} \mid \mu\right)$ as well as prior density $\pi(\mu)$ and estimate of posterior density $\hat{\pi}\left(\mu \mid \hat{\mu}_{10}\right)$ obtained with bandwidth $w=0,03$

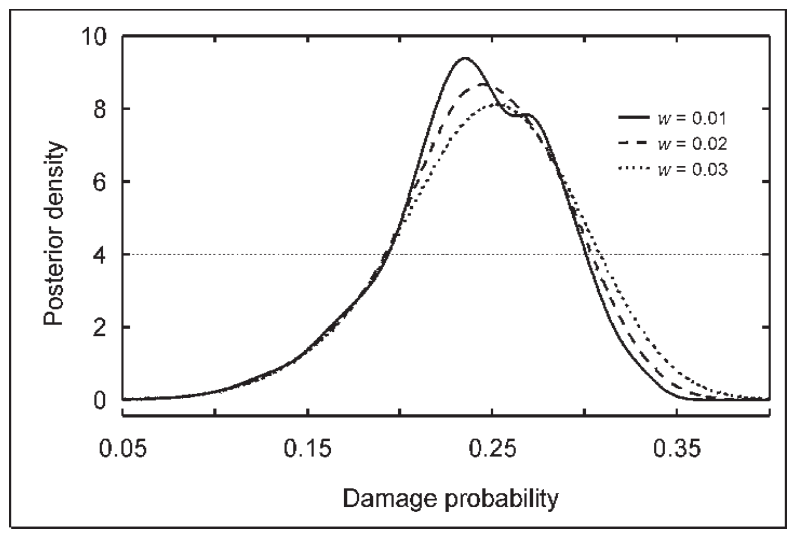

Fig 6. Posterior density of the damage probability at three values of bandwidth $w$
$P\left(D_{i} \mid A E\right)$

Three approximations $\hat{\pi}\left(\mu \mid \hat{\mu}_{10}\right)$ can be compared and so the influence of the bandwidth $w$ on the posterior density assessed by calculating approximate confidence intervals for each of the three $\hat{\pi}\left(\mu \mid \hat{\mu}_{10}\right)$. Table 2 shows three $90 \%$ confidence intervals at the three values of $w$. These intervals can be easily computed during numerical evaluation of $\hat{\pi}\left(\mu \mid \hat{\mu}_{10}\right)$.

The confidence intervals given in Table 2 can be compared with $90 \%$ bootstrap confidence interval computed using ten-element sample $\boldsymbol{p}$. The limits of the latter interval can be taken as the $5^{\text {th }}$ and the $95^{\text {th }}$ percentages of an ordered sample obtained from the bootstrap sample $\left(\hat{\mu}_{10,1}^{\prime}, \hat{\mu}_{10,2}^{\prime}, \ldots, \hat{\mu}_{10,1000}^{\prime}\right)$ [16]. The bootstrap confidence interval is ]0.0902, 0.304[. This interval is based on the new data only (ignores the prior knowledge). It is apparent that the width of the intervals given in Table 2 is considerably smaller than the one of the bootstrap confidence interval.

Table 2. Approximate $90 \%$ confidence intervals calculated for damage probability $P\left(D_{i} \mid A E\right)$ from posterior densities

\begin{tabular}{|c|c|c|}
\hline $\begin{array}{c}\text { Bandwidth } \\
w\end{array}$ & Constant $C\left(\hat{\mu}_{10}\right)$ & $\begin{array}{c}\text { Confidence } \\
\text { interval }\end{array}$ \\
\hline 0,01 & 1,2311 & ] 0,$158 ; 0,308[$ \\
\hline 0,02 & 1,2470 & ] 0,$159 ; 0,315[$ \\
\hline 0,03 & 1,2713 & ] 0,$160 ; 0,326[$ \\
\hline
\end{tabular}

The approximate confidence intervals given in Table 2 may be used for making a decision concerning whether the resistance of the wall panels is sufficient to resist damage $D_{i}$ or, alternatively, to design the panels for tolerable value of damage probability $P\left(D_{i} \mid A E\right)$.

\section{Conclusion}

The paper presents an approach to assessing damage to buildings due to accidental explosions (AEs) on the railway. The damage can be caused to nearby structures by the blast loading generated by AE. The attention is focussed on quantifying uncertainties related to both characteristics of AEs and damage from them. The damage is understood as structural failures caused by blast loading. Probabilities of these failures (damage probabilities) are taken as damage measures. The prediction of damage is realised estimating the damage probabilities.

A procedure of estimating damage probabilities has been proposed. The basis of the procedure is the application of a computer intensive method of applied statistics which is called the Bayesian bootstrap. This method is used for expressing estimates of the dam- 
age probabilities in terms of Bayesian posterior distributions. These distributions were treated as measures of epistemic uncertainty in damage probabilities.

Formally, the Bayesian bootstrap was applied to Bayesian inference using prior knowledge. It consists of mathematical models and historical data suitable for approximate prediction of loading from AE. Another part of this knowledge is new information. It is represented by a small-size sample of highly case-specific measurements of AE. The procedure is in a large measure automatic. It does not require any statistical derivations. Therefore, it can be applied in practical assessment of existing structures built in the vicinity of the railway and exposed to the hazard of AEs. The procedure can also be applied to specify safe distances between the railway and nearby future buildings.

\section{Refferences}

1. Baker, W. E.; Cox, P. A.; Westine, P. S. et al. Explosion Hazards and Evaluation. Amsterdam etc.: Elsevier, $1983.512 \mathrm{p}$.

2. Marshall, V. C. Major chemical hazards. Chichester: Ellis Horwood Ltd., 1987. 471 p.

3. Shao, J. \& Tu, D. The Jackknife and Bootstrap. New York etc.: Springer, 1995. 516 p.

4. Davison, A. C. \& Hinkley, D. V. Bootstrap methods and their application. Cambridge: Cambridge university press, 1998. $582 \mathrm{p}$.

5. Efron, B. \& Tibshirani, R. J. An introduction to the bootstrap. New York: Chapman \& Hall, 1993. 436 p.

6. Rubin, D. B. The Bayesian bootstrap. Annals of Statistics, Vol 9(1), 1981, p. 130-134.

7. Boos, D. D. \& Monahan, J. F. Bootstrap methods using prior information. Biometrica, Vol 73, No 1, p. 77-83.

8. Lo, A. A large sample study of the Bayesian bootstrap. Annals of Statistics, Vol 15, No 1, 1987, p. 360-375.

9. Lo, A. A Bayesian bootstrap for a finite population. Annals of Statistics, Vol 16, No 4, 1988, p. 1684-1695.

10. Lo, A. A Bayesian bootstrap for censored data. Annals of Statistics, Vol 21, No 1, 1993, p. 100-123.

11. Davison, A. C.; Hinkley, D. V. \& Worton, B. J. Bootstrap likelihoods. Biometrica, Vol 79, No 1, 1992, p. 113130.

12. Aven, T., Pörn, K. Expressing and interpreting the results of quantitative risk analyses. Review and discussion. Reliability Engineering \& System Safety, Vol 61, No 1, 1998, p. 3-10.

13. ENV 1991-2-7: Eurocode 1: Basis of design and actions on structures. Part 2-7: Accidental actions due to impact and explosions. Brussels: CEN, 1998. 257 p.

14. DNV-OS-A101. Offshore standard. Safety principles and arrangements. Høvik: Det Norske Veritas, 2001.
$433 \mathrm{p}$.

15. Vaidogas, E. R. Statistical resampling in assessing external threats to structures. In: C. Spitzer, U. Schmocker \& V. N. Dang (eds), Probabilistic safety assessment and management, Proc. PSAM 7-ESREL'04, Berlin, June 14-18, 2004, Vol 5, London etc.: Springer, p. 2921-2925.

16. Siu, N. O. \& Kelly, D. L. Bayesian parameter estimation in probabilistic risk assessment. Reliability Engineering \& System Safety, Vol 62, No 1, 1998, p. 89-116.

17. Vaidogas, E. R. Accidental explosions: Bayesian uncertainty handling in assessing damage to structures. In: A. Der Kiureghian, S. Madanat \& J. M. Pestana (eds), Application of statistics and probability in civil engineering; Proc. ICASP9, San Francisco, July 6-9, 2003, Rotterdam: Millpress, 2003, p. 191-198.

18. Twisdale, L. A. \& Vickery, P. J. Extreme wind risk assessment. In: C. (Raj) Sundararajan (ed.), Probabilistic structural mechanics handbook, New York etc.: Chapman\&Hall, 1995, p. 465-509.

19. Sarabandi, P. et al. Development of empirical building performance functions data from past earthquakes. In: A. Der Kiureghian, S. Madanat \& J. M. Pestana (eds), Application of statistics and probability in civil engineering; Proc. ICASP9, San Francisco, July 6-9, 2003, Rotterdam: Millpress, 2003, p. 629-635.

20. Congdon, P. Bayesian statistical modeling. Chichester: Wiley, 2001. $531 \mathrm{p}$.

21. Bernardo, J. M. \& Smith A. F. M. Bayesian theory. Chichester etc.: Wiley, 1994. 586 p.

22. Melchers, R. E. Structural reliability analysis and prediction. $2^{\text {nd }}$ ed. Chichester etc.: Wiley, 1999. 615 p.

23. Barnett, V. Sample survey. Principles and methods. London etc.: Edward Arnold, 1991. 273 p.

24. Apeland, S.; Aven, T.; Nilsen, T. Quantifying uncertainty under predictive, epistemic approach to risk analysis. Reliability Engineering \& System Safety, Vol 75, No 2, 2002, p. 93-102.

25. Tedesko, J. W.; McDougal, W. G. \& Ross, C. A. Structural dynamics. Theory and applications. Melno Parc, California: Addisin Wesley, 1999. 731 p.

26. Wharton, R. K.; Formby, S. A. \& Merrifield, R. Airblast TNT equivalence for a range of commercial blasting explosives. Journal of Hazardous Materials, Vol A79, No 1, 2000, p. 31-39.

27. Low, H. Y.; Hao, H. Reliability analysis of direct shear and flexural failure modes of RC slabs under explosive loading. Engineering Structures, Vol 24, No 2, 2002, p. 189-198. 